VANJA KORAĆ

Mathematical Institute SASA,

Kneza Mihaila 36/III,

Belgrade, Serbia,

e-mail:vanja@mi.sanu.ac.rs

ZORAN DAVIDOVAC

Mathematical Institute SASA,

Kneza Mihaila 36/III,

Belgrade, Serbia,

e-mail: zorandavidovac@mi.sanu.ac.rs
004.451.9.056.57

COBISS.SR-ID 254104844

Original research article

Received: March 07th 2017

Accepted: October $31^{\text {st }} 2017$

DRAGAN PRLJA

Institute for Comparative Law,

Terazije 41, Belgrade, Serbia,

e-mail:dprlja@yahoo.com

\title{
WINDOWS DEFAULT SERVICES VULNERABILITIES ASSESSMENT
}

\begin{abstract}
By using tools for analysing vulnerable services on the system it is possible to obtain valuable information about the system and the network in terms of protection. The research in this paper included 51 Windows operating systems. The collected information consists of a large amount of data about the presence of various network services on a system that present potential security flaws. Thus, the vulnerabilities of Windows operating systems that are installed by default are presented, with the aim of pointing out potential security vulnerabilities. These vulnerabilities or omissions can occur due to incorrectly configured services, well known bugs in the system or program, an outdated system and its services, and the use of poor protection in configuration. The aim of this assessment is to identify and correct accordingly all recognized security flaws (vulnerable services) on Windows systems installed by default.
\end{abstract}

\section{KEYWORDS: VULNERABILITY ANALYSIS, VULNERABILITY ASSESSMENT, WINDOWS VULNERABILITIES, OS VULNERABILITIES.}

In the system of protection, vulnerabilities can be in software, hardware, configuration and people (Grubor and Gotić 2012). ${ }^{1}$ In this research paper, the focus is on discovering the vulnerability of the

1 The article results from the project: Viminacium, Roman city and military camp - research of the material and no material culture of inhabitants by using the modern technologies of remote detection, geophysics, GIS, digitalization and $3 D$ visualization (no 47018), funded by The Ministry of Education, Science and Technological Development of the Republic of Serbia. operating system software, i.e., operating system services. By using tools for analysing vulnerable services on the system it is possible to obtain valuable information about the system and the network in terms of protection. As will be shown, the collected information will include a large number of data on the presence of various network services on the system that present potential security flaws. These omissions can occur due to incorrectly configured services, well known bugs in the system or 


\begin{tabular}{|l|l|}
\hline Source name & Web address of the source \\
\hline APPLE-SA (Apple Security Announce) & http://lists.apple.com/archives/security-announce \\
\hline BID & http://www.securityfocus.com/bid/ \\
\hline CERT CA & http://www.us-cert.gov/ncas/alerts/ \\
\hline CERT TA & http://www.us-cert.gov/ncas/alerts/ \\
\hline CERT-VN & http://www.kb.cert.org/vuls/ \\
\hline $\begin{array}{l}\text { CVE (Common Vulnerabilities and Expo- } \\
\text { sures) }\end{array}$ & $\begin{array}{l}\text { http://web.nvd.nist.gov/view/vuln/search i http://cve. } \\
\text { mitre.org/ }\end{array}$ \\
\hline $\begin{array}{l}\text { DEBIAN DSA (Debian Security An- } \\
\text { nounce) }\end{array}$ & http://www.debian.org/security/ \\
\hline $\begin{array}{l}\text { IAVM (Information Assurance Vulnerabil- } \\
\text { ity Management ) }\end{array}$ & http://iase.disa.mil/index2.html \\
\hline $\begin{array}{l}\text { MANDRAKE MDKSA (Mandrake Secu- } \\
\text { rity Announce) }\end{array}$ & $\begin{array}{l}\text { http://www.mandriva.com/en/support/security/adviso- } \\
\text { ries/ }\end{array}$ \\
\hline MS (Microsoft security) & http://technet.microsoft.com/en-us/security/dn481339 \\
\hline MSKB (Microsoft Knowledge Base) & http://support.microsoft.com/ \\
\hline NETBSD & ftp://ftp.netbsd.org/pub/NetBSD/security/advisories/ \\
\hline $\begin{array}{l}\text { OSVDB (Open Sourced Vulnerability } \\
\text { Database) }\end{array}$ & http://www.osvdb.org/ \\
\hline $\begin{array}{l}\text { OVAL (Open Vulnerability and Assess- } \\
\text { ment Language ) }\end{array}$ & http://oval.mitre.org/find/ \\
\hline $\begin{array}{l}\text { REDHAT RHSA (Redhat Security An- } \\
\text { nounce) }\end{array}$ & http://www.redhat.com/mailman/listinfo/rhsa-announce \\
\hline SANS & http://www.sans.org/critical-security-controls/ \\
\hline SECTRACK (SecurityTracker) & http://securitytracker.com/ \\
\hline SECUNIA & http://secunia.com/advisories \\
\hline SGI & ftp://patches.sgi.com/support/free/security/advisories/ \\
\hline nUSE SUSE-SA (SUSE Security An- & https://www.suse.com/support/security/advisories/ \\
\hline XF (X-force) & http://xforce.iss.net/ \\
\hline
\end{tabular}

Table 1 Sources that publish vulnerabilities on operating systems

program, an outdated system and its services, as well as the use of poor protection in configuration. The task of this test is to identify and correct all recognized security flaws (vulnerable services) on the systems that are installed by default. All relevant sources reporting vulnerabilities on systems are included and shown in Table 1.

The vulnerability problem can also be seen through the Symantec Vulnerability Report for
2011, according to which the number of vulnerabilities was $4989^{2}$, which means that almost 95 new vulnerabilities occur every week ${ }^{3}$. The peri-

2 This number is based on a large number of sources including mailing lists and recommendations of many producers of programs and equipment.

Source: http://www.symantec.com/threatreport/topic. jsp?id=vulnerability_trends\&aid=total_number_of_ vulnerabilities

3 ISource: http://www.symantec.com/threatreport/topic. jsp?id=vulnerability_trends\&aid=total_number_of_ 


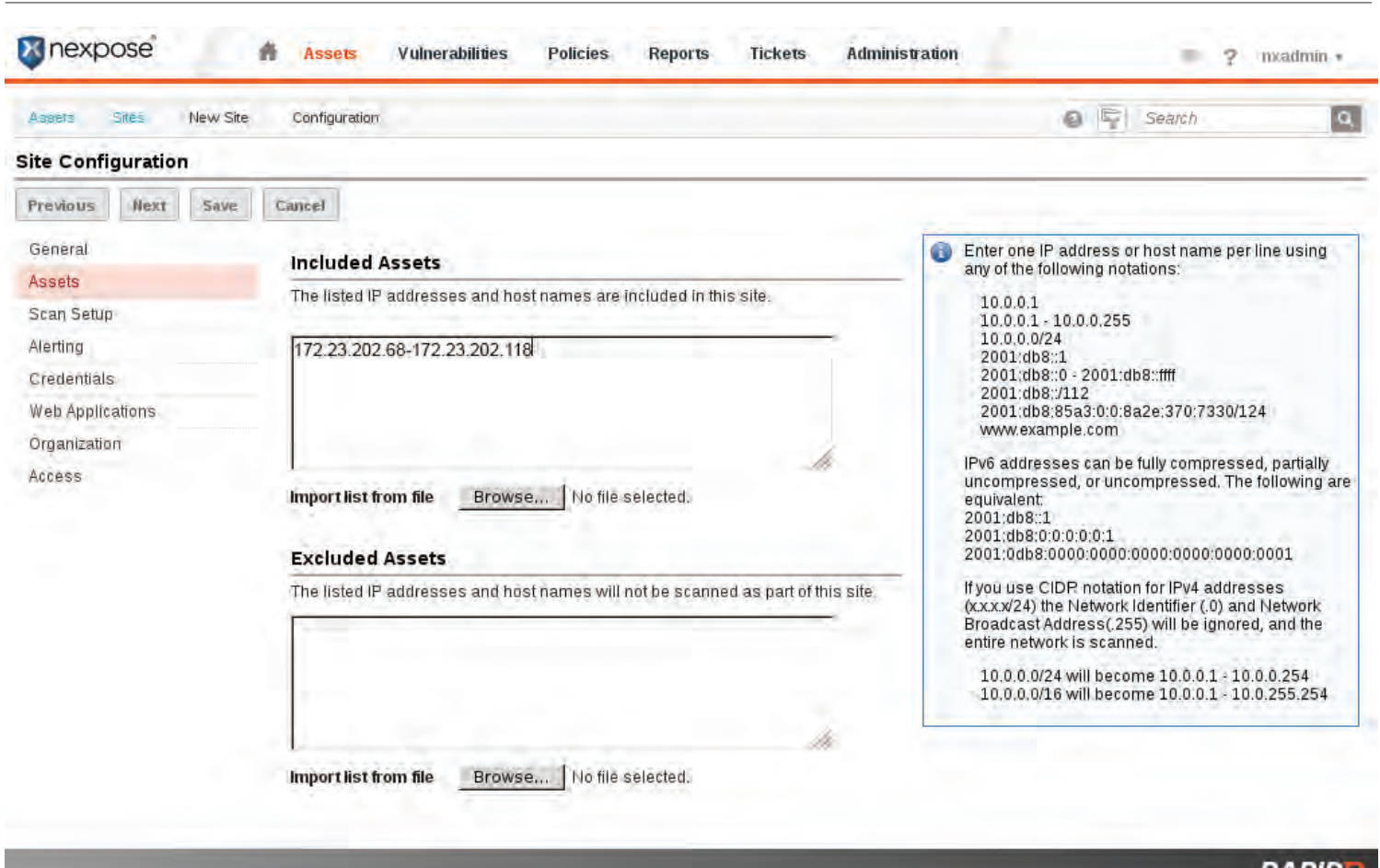

Fig. 1 Preparation of the Rapid 7 Nexpose tool for scanning of the service

od between publishing a vulnerability and applying a patch to a vulnerable program or service on the system is a critical period. The tool used for this work is called RAPID 7 Nexpose (Figure 1). With this tool, it is possible to perform planned and selective testing over network services, servers within an organization, and key services in the search for vulnerabilities that can be misused by attackers. In practice, corrective measures are proposed after system scanning. The total number of operating systems covered by this survey is 51 Windows operating systems.

The virtual environment for research purposes with the operating systems shown in the tables (Table 3., Table 4.) was realized within the Vmware ESX 5.1.0 platform, the IBM x3650 M3 server and the EMC VNX5300 system, thereby achieving a centralized consolidation of all virtual computer systems intended for testing. Thus a stable platform for effective vulnerability testing with a high level of security was provided.

The virtual environment platform is VMWare ESXi 5.1.0, which is implemented on vulnerabilities the IBMx3650 server (Figure 2.) and the EMC VNX5300 Storage system.

Server specifications for the IBMx3650 M3:

- 8 CPU Cores (2 x 4C Xeon E5620 80W, 2.4 GHZ 12MB cache

- 56 GB RAM PC3L-10600 ECC DDR3 1333

MHz memory

- 4x IBM 900 GB SAS HDD

- ServeRAID M5014 SAS/SATA controller

- IBM 460W Redundant Power Supply

- IBM UltraSlim Enhanced SATA Multi-Burner

The EMC VNX5300 storage system, with mounted virtual machines for testing purposes, consists of an Intel Xeon 5600 processor, with 16GB cache memory, $8 \times$ 8Gbit FC port, $8 \times$ 1GbE port, 25 x 600GB SAS 15k RPM, 25 x 2TB NL- SAS 7k RPM drives, 5 x 100GB FAST Cache Flash drive, rack cabinet VNX-40U, support for additional capacity expansion, support for CIFS, NFS, iSCSI and FC protocols, Local Protection Suite licenses, Security \& Compliance Suite licenses, redundant power supplies. Table 2 contains a more detailed specification of this system.

The following tables show the operating sys- 


\begin{tabular}{|l|}
\hline VNX5300 CONTROL STATION - EMC RACK \\
\hline 2 x 1GBE DM MODULE 4 PORT FOR VNX5300 \\
\hline VNX5300 ADD ON DM+FC SLIC-EMC RACK \\
\hline VNX5300 DME: 1 D M+FC SLIC-EMC RACK \\
\hline VNX5300 DPE; 15X3.5 DRIVES EMC RACK 8X600GB \\
15K \\
\hline 3 x 3U DAE WITH 15X3.5 INCH DRIVE SLOTS WITH \\
RACK \\
\hline 5 X 100GB FAST CACHE FLSH 15X3.5IN DPE/DAE \\
\hline 17X 600GB 15K SAS DISK DRIVE \\
\hline VNX 40U RACK WITH CONSOLE \\
\hline EMC VNX5300 4 PORT 8G FC IO MODULE PAIR \\
\hline ADDITIONAL 8 G FC SFP FOR VNX 51/53 \\
\hline RACK-40U-60 PWR CORD IEC 309 \\
\hline EMC DOCUMENTATION KIT FOR VNX5300 \\
\hline SECURITY \& COMPLIANCE SUITE FOR VNX5300 \\
\hline LOCAL PROTECTION SUITE FOR VNX5300 \\
\hline FAST CACHE FOR VNX5300 \\
\hline BASE FILE LICENSE (CIFS AND FTP) FOR VNX5300 \\
\hline ADV FILE LICENSE (NFS; MPFS AND PNFS) FOR \\
\hline VNX5300 \\
\hline UNISPHERE UNIFIED \& VNX OE VNX5300 \\
\hline 25 x 2TB 7200RPM 6GB SAS DISK DRIVE \\
\hline EMC 2ND OPTIONAL SPS \\
\hline EMC ENHANCED SOFTWARE SUPPORT \\
\hline
\end{tabular}

Table 2 Specification of the EMC VNX 5300 storage system

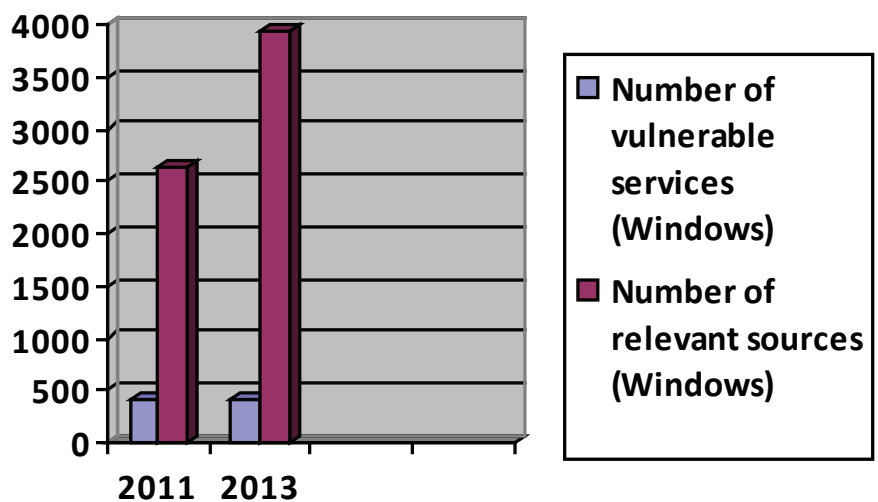

Graph 1 Presentation of vulnerable services found on Windows OS with the number of relevant sources reporting vulnerabilities in 2011 and 2013 
(2)

File Edit View Inventory Administration Plug-ins Help

(4) A Home D in Inventory D

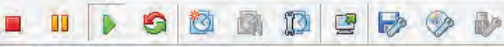

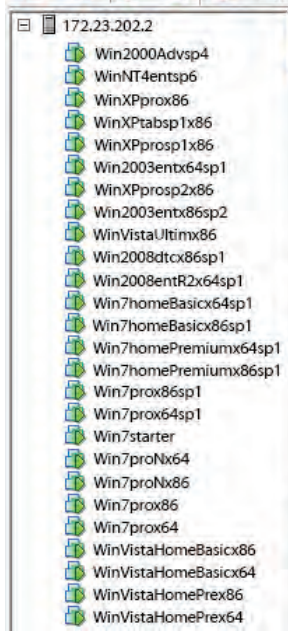

Getting Started Summary Vesource Allocation) Performance) (Events) Console) Perrmissions

What is a Virtual Machine?

A virtual machine is a software computer that, like a physical computer, runs an operating system and applications. An operating system installed on a virtual machine is called a guest operating system.

Because every virtual machine is an isolated computing environment you can use virtual machines as desktop or workstation environments, as testing environments, or to consolidate server applications.

Virtual machines run on hosts. The same host can run many virtual machines.

\section{Basic Tasks}

- Shut down the virtual machine

II] Suspend the virtual machine

Edit virtual machine settings

Fig. 1 Part of virtual machines prepared for vulnerable systems scanning

\begin{tabular}{|c|c|c|c|c|c|c|c|c|c|c|}
\hline Xnexpose & $\boldsymbol{H}$ & Assets & Vulnerabilities & Reports & Tickets & \multicolumn{2}{|c|}{ Administration } & \multicolumn{2}{|r|}{00 ? } & nxadmin • \\
\hline Assets & Windows os & Seans & Full audit & & & & & $9 \sqrt{8}$ & Search & Q \\
\hline Scan Progress & & & & & & & & & & $-x$ \\
\hline Scan Type & Started & & & Assets Vuinerabilities & Elapse & & Statu & & & Scan Log \\
\hline Manual & Sun 24 Nov $2 C$ & 2013 07:33:0 & AM CET & 417 & 3 minu & tes & Comp & pleted successfully & & Q \\
\hline Discovered Assets & & & & & & & & & & 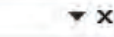 \\
\hline Address & & Name & & Operating System & & Vulnerabilities & & Scan Duration & Scan Status & \\
\hline $172,23,202,110$ & & WINZ20DOOAD & AICE & Microsoft Windows 2000 & & & 19 & 18 seconds & Completed & \\
\hline 172.23 .202 .101 & & WININT4 & & Microsoft Windows NT 4.0 & & & 22 & 3 minutes & Completed & \\
\hline $172,23,202.105$ & & WIINXPPPO & & Microsoft Windows XP & & & 15 & 48 seconds & Completed & \\
\hline $172,23.202 .109$ & & WIINXPTABL & $T \times 86$ & Microsoft Windows XP & & & 15 & 48 seconds & Completed & \\
\hline $172,23.202 .106$ & & WINXXPSP1 & & Microsoft Windows XP & & & 15 & 48 seconds & Completed & \\
\hline 172.23 .202 .111 & & WIII2003EI: & k64 & Microsoft Windows Server 2003 & 3 SP1 & & 14 & 22 seconds & Completed & \\
\hline $172,23,202,107$ & & WINXPSPZ & & Microsoft Windows XP & & & 11 & 24 seconds & Completed & \\
\hline $172,23.202 .112$ & & WIII2003E| & SP2 & Microsoft Windows Server 2003 & $3 \mathrm{SP} 2$ & & 9 & 22 seconds & Completed & \\
\hline 172.23 .202 .113 & & VISTAXE6UL & & $\begin{array}{l}\text { Microsoft Windows Vista Ultimat } \\
\text { Edition }\end{array}$ & & & 8 & 22 seconds & Completed & \\
\hline 172.23 .202 .117 & & 2008DATAC & 16SPI & $\begin{array}{l}\text { Microsoft Windows Server } 2008 \\
\text { Datacenter Edition }\end{array}$ & & & 7 & 22 seconds & Completed & \\
\hline 172.23 .202 .115 & & WHITULTX8 & SP1 & $\begin{array}{l}\text { Microsoft Windows } 7 \text { Ultimate Ec } \\
\text { SP1 }\end{array}$ & Edition & & 4 & 22 seconds & Completed & \\
\hline 172.23 .202 .116 & & WIHA7ULTX6 & & Microsoft Windows 7 Ultimate $\mathrm{Ec}$ & Edition & & 4 & 22 seconds & Completed & \\
\hline $172,23.202 .108$ & & WIINXPSP3 & & Microsoft Windows XP & & & 4 & 23 seconds & Completed & \\
\hline 17223.202 .118 & & 2008EIITH2 & 64SP1 & $\begin{array}{l}\text { Microsoft Windows Server } 2008 \\
\text { Enterprise Edition SP1 }\end{array}$ & 8R2, & & 4 & 22 seconds & Completed & \\
\hline 172.23 .202 .103 & & WINGESE & & $\begin{array}{l}\text { Microsoft Windows } 98 \text { SE (no se } \\
\text { pack) }\end{array}$ & service & & 3 & 11 seconds & Completed & \\
\hline $172,23.202 .104$ & & WIIIME & & Microsoft Windows 2000 SP2 & & & 2 & 16 seconds & Completed & \\
\hline $172,23.202 .102$ & & & & $\begin{array}{l}\text { Microsoft Windows for Workgrou } \\
\text { 3.11, Windows NT } 3.51 \text { SP0 - SP } \\
\text { Windows } 95\end{array}$ & $\begin{array}{l}\text { sups } \\
\text { SP5, or }\end{array}$ & & 2 & 47 seconds & Completed & \\
\hline
\end{tabular}

Fig. 3 Completed Rapid 7 Nexpose tool scanning of vulnerable services on Windows platforms 


\begin{tabular}{|c|c|c|c|}
\hline No. & Operating System & Computer Name & IP Address \\
\hline 1. & Windows nt 4 enterprise sp6 & NT4entsp6 & 172.23 .202 .101 \\
\hline 2. & Windows 95 OSR 2.5 & Win95OSR & 172.23.202.102 \\
\hline 3 & Windows 98 se & WIN98SE & 172.23.202.103 \\
\hline 4. & Windows ME & WINME & 172.23.202.104 \\
\hline 5. & Windows XP pro x86 & WINXPprox86 & 172.23.202.105 \\
\hline 6. & Windows xp pro sp1 x86 & WINXPproSP1x86 & 172.23.202.106 \\
\hline 7. & Windows xp pro sp2 x86 & WINXPproSP2x86 & 172.23.202.107 \\
\hline 8. & Windows xp pro sp3 x86 & WINXPproSP3x86 & 172.23.202.108 \\
\hline 9. & Windows xp tablet pc SP1 & WINXPTabX86sp1 & 172.23.202.109 \\
\hline 10. & Windows 2000 advanced server sp4 & WIN2000ADVsp4 & 172.23.202.110 \\
\hline 11. & Windows server 2003 Enterprise x64 SP1 & WIN2003ENX64sp1 & 172.23.202.111 \\
\hline 12. & Windows Server 2003 Enterprise x86 sp2 & WIN2003ENSP2x86 & 172.23.202.112 \\
\hline 13. & Windows Vista ultimate x86 & VISTAx86ULT & 172.23.202.113 \\
\hline 14. & Windows Vista Ultimate SP2 x86 & VISTAx86ULTSP2 & 172.23.202.114 \\
\hline 15. & Windows 7 ultimate x86 sp1 & WIN7x86ULTSP1 & 172.23.202.115 \\
\hline 16. & Windows 7 ultimate x64 & WIN7x64ULT & 172.23.202.116 \\
\hline 17. & $\begin{array}{l}\text { Windows } 2008 \text { server datacenter x86 SP1 } \\
\text { (kernel as Windows Vista ultim sp2) }\end{array}$ & 2008DTCX86SP1 & 172.23.202.117 \\
\hline 18. & $\begin{array}{l}\text { Windows } 2008 \text { enterprise x64 server R2 } \\
\text { SP1 update June 2011SP1 (kernel as Win- } \\
\text { dows 7) }\end{array}$ & 2008entR2X64SP1 & 172.23.202.118 \\
\hline 19. & Windows 7 Home Basic SP1 x64 & WIN7x64HoBaSp1 & 172.23.202.100 \\
\hline 20. & Windows 7 Home Basic SP1 x86 & WIN7x86HoBaSp1 & 172.23.202.99 \\
\hline 21 & Windows 7 Home Premium SP1 x64 & WIN7x64HoPreSp1 & 172.23.202.98 \\
\hline 22. & Windows 7 Home Premium SP1 x86 & WIN7x86HoPreSp1 & 172.23.202.97 \\
\hline 23. & Windows 7 Professional SP1 x64 & WIN7x64ProSp1 & 172.23.202.96 \\
\hline 24. & Windows 7 Professional SP1 x86 & WIN7x86ProSp1 & 172.23.202.95 \\
\hline 25. & Windows 7 starter & WIN7starter & 172.23.202.94 \\
\hline 26. & Windows 7 Professional N x64 & WIN7ProNx64 & 172.23.202.93 \\
\hline 26. & Windows 7 Professional N x86 & WIN7ProNx86 & 172.23.202.92 \\
\hline 28. & Windows 7 Professional x64 & WIN7Prox64 & 172.23.202.91 \\
\hline 29. & Windows 7 Professional x86 & WIN7Prox86 & 172.23.202.90 \\
\hline 30. & Windows Vista Home Basic x86 & VISTAx86НоВа & 172.23.202.89 \\
\hline 31. & Windows Vista Home Basic x64 & VISTAx64HoBa & 172.23 .202 .88 \\
\hline 32. & Windows Vista Home Premium x86 & VISTAx86HoPre & 172.23.202.87 \\
\hline 33. & Windows Vista Home Premium x64 & VISTAx64HoPre & 172.23.202.86 \\
\hline 34. & Windows Vista Business x86 & VISTAx86Bsn & 172.23.202.85 \\
\hline
\end{tabular}




\begin{tabular}{|l|l|l|l|}
\hline 35. & Windows Vista Business x64 & VISTAx64Bsn & 172.23 .202 .84 \\
\hline 36. & Windows Vista Ultimate x64 & VISTAx64ULT & 172.23 .202 .83 \\
\hline 37. & Windows Vista Home Basic x86 SP2 & VISTAx86HoBaSp2 & 172.23 .202 .82 \\
\hline 38. & Windows Vista Home Basic x64 SP2 & VISTAx64HoBaSp2 & 172.23 .202 .81 \\
\hline 39. & Windows Vista Business x86 SP2 & VISTAx86BsnSp2 & 172.23 .202 .80 \\
\hline 40. & Windows Vista Business x64 SP2 & VISTAx64BsnSp2 & 172.23 .202 .79 \\
\hline 41. & Windows Vista Home Premium x86 SP2 & VISTAx86HoPrSp2 & 172.23 .202 .78 \\
\hline 42. & Windows Vista Home Premium x64 SP2 & VISTAx64HoPrSp2 & 172.23 .202 .77 \\
\hline 43. & Windows 2000 server Sp4 & Win2000srv & 172.23 .202 .76 \\
\hline 44. & Windows server 2003 Enterprise x86 SP1 & WIN2003ENX86sp1 & 172.23 .202 .75 \\
\hline 45. & Windows server 2003 Standard x86 SP1 & WIN2003StX86sp1 & 172.23 .202 .74 \\
\hline 46. & Windows server 2003 Standard x64 SP1 & WIN2003StX64sp1 & 172.23 .202 .73 \\
\hline 47. & Windows Server 2003 Enterprise x64 SP2 & WIN2003ENSP2x64 & 172.23 .202 .72 \\
\hline 48. & Windows server 2003 Standard x86 SP2 & WIN2003StX86sp2 & 172.23 .202 .71 \\
\hline 49. & Windows server 2003 Standard x64 SP2 & WIN2003StX64sp2 & 172.23 .202 .70 \\
\hline 50. & Windows XP pro sp1 x64 & WINXPproSP1x64 & 172.23 .202 .69 \\
\hline 51 & Windows 2008 server Enterprise x86 SP1 & 2008EntX86SP1 & 172.23 .202 .68 \\
\hline
\end{tabular}

Table 3 Windows operating systems

tems included in vulnerability scanning with the RAPID 7 Nexpose ${ }^{4}$ tool:

Table 3 lists the versions of Windows operating systems, the names of the computers with the IP addresses that are included in the scan, by the Rapid7 Nexpose tool. By default, Windows operating systems are installed without added services.

Table 4 shows an overview of the total number of detected vulnerabilities and their relevant sources related to Windows operating systems in 2011 and 2013.

In Table 5 the number of vulnerabilities on Windows OS is presented with detailed review according to severity (Critical $-\mathrm{Cr}$, Serious $-\mathrm{Se}$, Moderate - Mo, Total - To)

The testing was carried out on 51 Windows operating system. U 2011, 144 unique vulnerabilities were found, and at the level of all scanned Windows systems, the total number is 414 vulnerabilities (Table 5, Graph 1). Out of this number, 79 critical, 43 serious and 22 moderate vulnera-

4 https://www.rapid7.com/products/nexpose/ bilities were found (Graph 2.), respectively considering all scanned systems together 211 critical, 117 serious and 86 moderate vulnerabilities were found (Table 5). Critical vulnerabilities require emergency intervention (Korać 2014). They can be relatively easy abused by a malicious attacker and by their exploitation it is possible to obtain total control over the affected computer system. Serious vulnerabilities are more difficult to exploit and in most cases they can not provide simultaneous access to the system. Concerning moderate vulnerabilities, they most often provide information that attackers can use to organize future attacks on computer systems in the network. Moderate vulnerabilities must also be resolved in a timely manner, but they are not as urgent as the two previously described. As already mentioned, when the computing systems are viewed individually, 211 critical, 117 serious and 86 moderate vulnerabilities were found in total. Critical vulnerabilities were found in a total of 34 computer systems and they are most susceptible to attack 


\begin{tabular}{|c|c|c|c|c|c|c|c|}
\hline$\dot{8}$ & 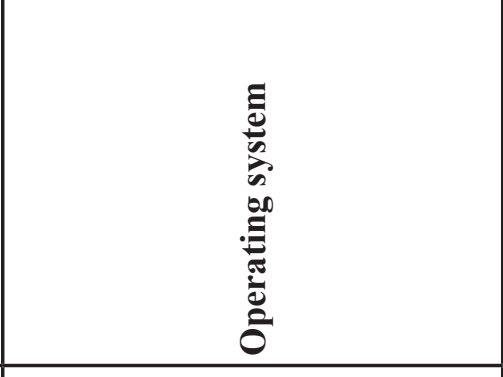 & 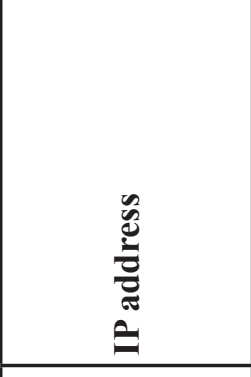 & 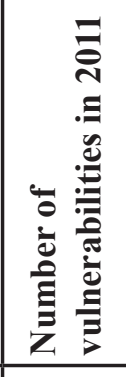 & 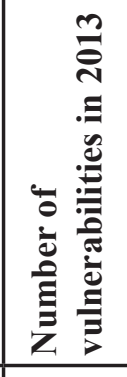 & : & 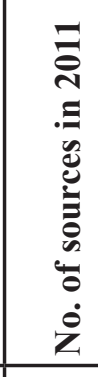 & 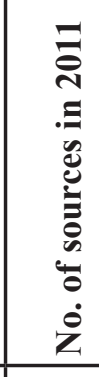 \\
\hline 1. & Windows nt 4 enterprise sp6 & 172.23.202.101 & 21 & 22 & 1 & 97 & 139 \\
\hline 2. & Windows 95 OSR 2.5 & 172.23.202.102 & 2 & 2 & 0 & 23 & 27 \\
\hline 3 & Windows 98 SE & 172.23.202.103 & 3 & 3 & 0 & 22 & 27 \\
\hline 4. & Windows ME & 172.23.202.104 & 2 & 2 & 0 & 23 & 23 \\
\hline 5. & Windows XP pro x86 & 172.23.202.105 & 15 & 15 & 0 & 174 & 247 \\
\hline 6. & Windows XP pro sp1 x86 & 172.23.202.106 & 15 & 15 & 0 & 174 & 248 \\
\hline 7. & Windows XP pro sp2 x86 & 172.23.202.107 & 11 & 11 & 0 & 54 & 54 \\
\hline 8. & Windows XP pro sp3 x86 & 172.23.202.108 & 4 & 4 & 0 & 9 & 10 \\
\hline 9. & Windows XP tablet pc SP1 x86 & 172.23.202.109 & 15 & 15 & 0 & 174 & 248 \\
\hline 10. & $\begin{array}{l}\text { Windows } 2000 \text { advanced server } \\
\text { sp4 }\end{array}$ & 172.23.202.110 & 18 & 19 & 1 & 225 & 54 \\
\hline 11. & $\begin{array}{l}\text { Windows server } 2003 \text { Enterprise } \\
\text { x64 SP1 }\end{array}$ & 172.23.202.111 & 14 & 14 & 0 & 89 & 10 \\
\hline 12. & $\begin{array}{l}\text { Windows Server } 2003 \text { Enter- } \\
\text { prise x86 sp2 }\end{array}$ & 172.23.202.112 & 9 & 9 & 0 & 41 & 248 \\
\hline 13. & Windows Vista ultimate x86 & 172.23.202.113 & 8 & 8 & 0 & 53 & 307 \\
\hline 14. & $\begin{array}{l}\text { Windows Vista Ultimate SP2 } \\
\text { x86 }\end{array}$ & 172.23.202.114 & 7 & 7 & 0 & 24 & 135 \\
\hline 15. & Windows 7 ultimate x86 sp1 & 172.23.202.115 & 4 & 4 & 0 & 8 & 73 \\
\hline 16. & Windows 7 ultimate x64 & 172.23.202.116 & 4 & 4 & 0 & 8 & 57 \\
\hline 17. & $\begin{array}{l}\text { Windows } 2008 \text { server datacenter } \\
\text { x86 SP1 (kernel as Windows } \\
\text { Vista ultim sp2) }\end{array}$ & 172.23 .202 .117 & 7 & 7 & 0 & 24 & 42 \\
\hline 18. & $\begin{array}{l}\text { Windows } 2008 \text { enterprise x64 } \\
\text { server R2 SP1 update June } \\
\text { 2011SP1 (kerenel as Windows } \\
\text { 7) }\end{array}$ & 172.23.202.118 & 4 & 4 & 0 & 8 & 8 \\
\hline 19. & $\begin{array}{l}\text { Windows } 7 \text { Home Basic SP1 } \\
\text { x64 }\end{array}$ & 172.23.202.100 & 4 & 4 & 0 & 8 & 8 \\
\hline 20. & $\begin{array}{l}\text { Windows } 7 \text { Home Basic SP1 } \\
\text { x86 }\end{array}$ & 172.23.202.99 & 4 & 4 & 0 & 8 & 42 \\
\hline 21 & $\begin{array}{l}\text { Windows } 7 \text { Home Premium SP1 } \\
\text { x64 }\end{array}$ & 172.23.202.98 & 4 & 4 & 0 & 8 & 8 \\
\hline
\end{tabular}




\begin{tabular}{|c|c|c|c|c|c|c|c|}
\hline$\dot{z}$ & 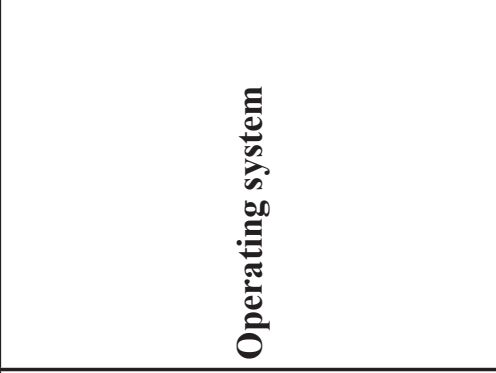 & 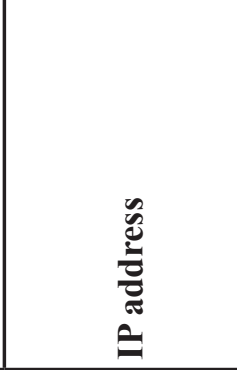 & 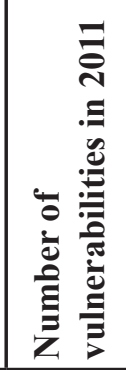 & 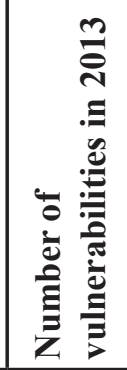 & 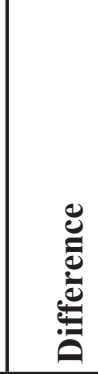 & 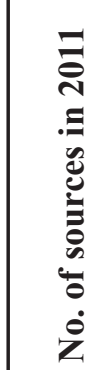 & 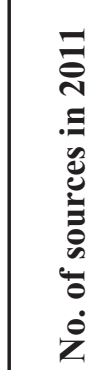 \\
\hline 22. & $\begin{array}{l}\text { Windows } 7 \text { Home Premium SP1 } \\
\text { x86 }\end{array}$ & 172.23.202.97 & 4 & 4 & 0 & 8 & 8 \\
\hline 23. & $\begin{array}{l}\text { Windows } 7 \text { Professional SP1 } \\
\text { x64 }\end{array}$ & 172.23.202.96 & 4 & 4 & 0 & 8 & 8 \\
\hline 24. & $\begin{array}{l}\text { Windows } 7 \text { Professional SP1 } \\
\text { x86 }\end{array}$ & 172.23.202.95 & 4 & 4 & 0 & 8 & 8 \\
\hline 25. & Windows 7 starter & 172.23.202.94 & 4 & 4 & 0 & 8 & 8 \\
\hline 26. & Windows 7 Professional N x64 & 172.23.202.93 & 4 & 4 & 0 & 8 & 8 \\
\hline 26. & Windows 7 Professional N x86 & 172.23.202.92 & 4 & 4 & 0 & 8 & 8 \\
\hline 28. & Windows 7 Professional x64 & 172.23.202.91 & 4 & 4 & 0 & 8 & 8 \\
\hline 29. & Windows 7 Professional x86 & 172.23 .202 .90 & 4 & 4 & 0 & 8 & 8 \\
\hline 30. & Windows Vista Home Basic x86 & 172.23.202.89 & 8 & 8 & 0 & 53 & 57 \\
\hline 31. & Windows Vista Home Basic x64 & 172.23.202.88 & 8 & 8 & 0 & 53 & 57 \\
\hline 32. & $\begin{array}{l}\text { Windows Vista Home Premium } \\
\text { x86 }\end{array}$ & 172.23.202.87 & 8 & 8 & 0 & 53 & 57 \\
\hline 33. & $\begin{array}{l}\text { Windows Vista Home Premium } \\
\text { x64 }\end{array}$ & 172.23.202.86 & 8 & 8 & 0 & 53 & 57 \\
\hline 34. & Windows Vista Business x86 & 172.23 .202 .85 & 8 & 8 & 0 & 53 & 57 \\
\hline 35. & Windows Vista Business x64 & 172.23.202.84 & 8 & 8 & 0 & 53 & 57 \\
\hline 36. & Windows Vista Ultimate x64 & 172.23 .202 .83 & 8 & 8 & 0 & 53 & 57 \\
\hline 37. & $\begin{array}{l}\text { Windows Vista Home Basic x86 } \\
\text { SP2 }\end{array}$ & 172.23.202.82 & 7 & 7 & 0 & 24 & 42 \\
\hline 38. & $\begin{array}{l}\text { Windows Vista Home Basic x64 } \\
\text { SP2 }\end{array}$ & 172.23.202.81 & 7 & 7 & 0 & 24 & 42 \\
\hline 39. & $\begin{array}{l}\text { Windows Vista Business x86 } \\
\text { SP2 }\end{array}$ & 172.23 .202 .80 & 7 & 7 & 0 & 24 & 42 \\
\hline 40. & $\begin{array}{l}\text { Windows Vista Business x64 } \\
\text { SP2 }\end{array}$ & 172.23.202.79 & 7 & 7 & 0 & 24 & 42 \\
\hline 41. & $\begin{array}{l}\text { Windows Vista Home Premium } \\
\text { x86 SP2 }\end{array}$ & 172.23.202.78 & 7 & 7 & 0 & 24 & 42 \\
\hline 42. & $\begin{array}{l}\text { Windows Vista Home Premium } \\
\text { x64 SP2 }\end{array}$ & 172.23.202.77 & 7 & 7 & 0 & 24 & 42 \\
\hline 43. & Windows 2000 server Sp4 & 172.23 .202 .76 & 18 & 19 & 1 & 225 & 307 \\
\hline
\end{tabular}




\begin{tabular}{|c|c|c|c|c|c|c|c|}
\hline$\dot{\dot{z}}$ & 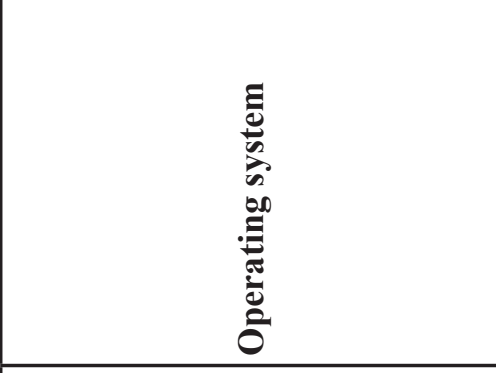 & 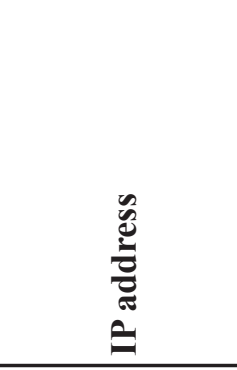 & 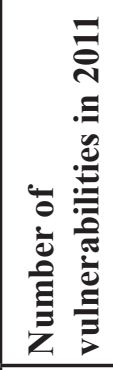 & 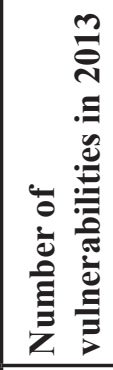 & 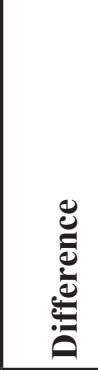 & 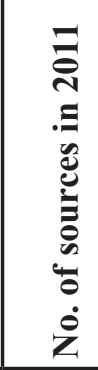 & 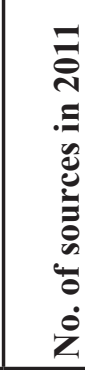 \\
\hline 44. & $\begin{array}{l}\text { Windows server } 2003 \text { Enterprise } \\
\text { x86 SP1 }\end{array}$ & 172.23.202.75 & 14 & 14 & 0 & 89 & 135 \\
\hline 45. & $\begin{array}{l}\text { Windows server } 2003 \text { Standard } \\
\text { x86 SP1 }\end{array}$ & 172.23.202.74 & 14 & 14 & 0 & 89 & 135 \\
\hline 46. & $\begin{array}{l}\text { Windows server } 2003 \text { Standard } \\
\text { x64 SP1 }\end{array}$ & 172.23.202.73 & 14 & 14 & 0 & 89 & 135 \\
\hline 47. & $\begin{array}{l}\text { Windows Server } 2003 \text { Enter- } \\
\text { prise x64 SP2 }\end{array}$ & 172.23.202.72 & 9 & 9 & 0 & 41 & 73 \\
\hline 48. & $\begin{array}{l}\text { Windows server } 2003 \text { Standard } \\
\text { x86 SP2 }\end{array}$ & 172.23.202.71 & 9 & 9 & 0 & 41 & 73 \\
\hline 49. & $\begin{array}{l}\text { Windows server } 2003 \text { Standard } \\
\text { x64 SP2 }\end{array}$ & 172.23.202.70 & 9 & 9 & 0 & 41 & 73 \\
\hline 50. & Windows XP pro sp1 x64 & 172.23.202.69 & 15 & 15 & 0 & 174 & 248 \\
\hline 51 & $\begin{array}{l}\text { Windows } 2008 \text { server Enterprise } \\
\text { x86 SP1 }\end{array}$ & 172.23.202.68 & 7 & 7 & 0 & 24 & 42 \\
\hline \multicolumn{3}{|c|}{ TOTAL } & 414 & 417 & 3 & 2646 & 3951 \\
\hline
\end{tabular}

Table 4 Windows vulnerabilities and their sources from 2011 and 2013

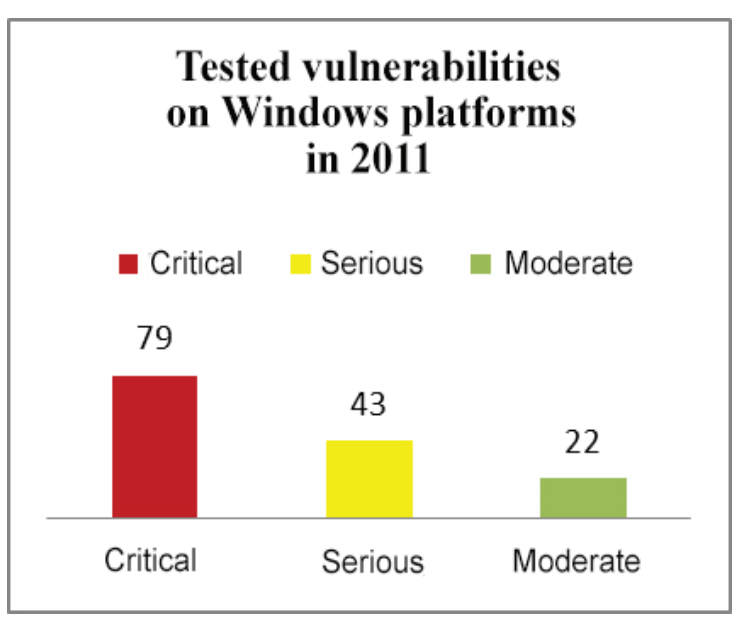

Graph 2 Found vulnerabilities on tested Windows OS in 2011

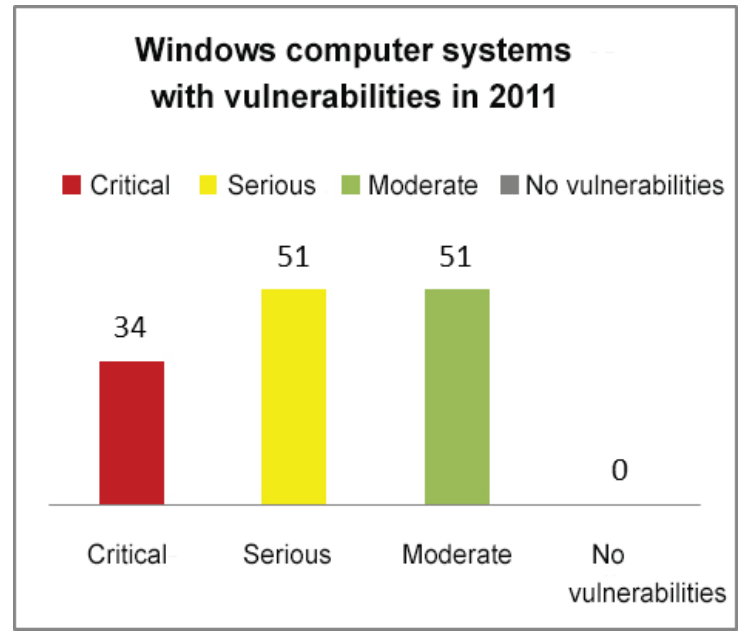

Graph 3 Number of Windows computing systems by severity of vulnerability in 2011 


\begin{tabular}{|c|c|c|c|c|c|c|c|c|c|c|}
\hline \multirow[t]{2}{*}{ No. } & \multirow[t]{2}{*}{ Operating system } & \multirow[t]{2}{*}{ IP Address } & \multicolumn{4}{|c|}{$\begin{array}{c}\text { Number of } \\
\text { vulnerabilities in } \\
2011 \\
\end{array}$} & \multicolumn{4}{|c|}{$\begin{array}{c}\text { Number of } \\
\text { vulnerabilities in } \\
2013 \\
\end{array}$} \\
\hline & & & $\mathrm{Cr}$ & $\mathrm{Se}$ & Mo & To & $\mathbf{C r}$ & Se & Mo & To \\
\hline 1. & Windows nt 4 enterprise sp6 & 172.23.202.101 & 9 & 11 & 1 & 21 & 10 & 11 & 1 & 22 \\
\hline 2. & Windows 95 OSR 2.5 & 172.23 .202 .102 & 0 & 1 & 1 & 2 & 0 & 1 & 1 & 2 \\
\hline 3 & Windows 98 se & 172.23 .202 .103 & 0 & 1 & 2 & 3 & 0 & 1 & 2 & 3 \\
\hline 4. & Windows ME & 172.23 .202 .104 & 0 & 1 & 1 & 2 & 0 & 1 & 1 & 2 \\
\hline 5. & Windows XP pro x86 & 172.23.202.105 & 11 & 3 & 1 & 15 & 11 & 3 & 1 & 15 \\
\hline 6. & Windows xp pro sp1 x86 & 172.23.202.106 & 11 & 3 & 1 & 15 & 11 & 3 & 1 & 15 \\
\hline 7. & Windows xp pro sp2 x86 & 172.23 .202 .107 & 8 & 2 & 1 & 11 & 8 & 2 & 1 & 11 \\
\hline 8. & Windows xp pro sp3 x86 & 172.23.202.108 & 1 & 2 & 1 & 4 & 1 & 2 & 1 & 4 \\
\hline 9. & Windows xp tablet pc SP1 & 172.23.202.109 & 11 & 3 & 1 & 15 & 11 & 3 & 1 & 15 \\
\hline 10. & Windows 2000 advanced server sp4 & 172.23 .202 .110 & 13 & 1 & 4 & 18 & 14 & 4 & 1 & 19 \\
\hline 11. & $\begin{array}{l}\text { Windows server } 2003 \text { Enterprise } \\
\text { x64 SP1 }\end{array}$ & 172.23 .202 .111 & 10 & 3 & 1 & 14 & 10 & 3 & 1 & 14 \\
\hline 12. & $\begin{array}{l}\text { Windows Server } 2003 \text { Enterprise } \\
\text { x86 sp2 }\end{array}$ & 172.23 .202 .112 & 6 & 2 & 1 & 9 & 6 & 2 & 1 & 9 \\
\hline 13. & Windows Vista ultimate x86 & 172.23.202.113 & 4 & 2 & 2 & 8 & 4 & 2 & 2 & 8 \\
\hline 14. & Windows Vista Ultimate SP2 x86 & 172.23 .202 .114 & 3 & 2 & 2 & 7 & 3 & 2 & 2 & 7 \\
\hline 15. & Windows 7 ultimate x86 sp1 & 172.23 .202 .115 & 0 & 2 & 2 & 4 & 0 & 2 & 2 & 4 \\
\hline 16. & Windows 7 ultimate x64 & 172.23.202.116 & 0 & 2 & 2 & 4 & 0 & 2 & 2 & 4 \\
\hline 17. & $\begin{array}{l}\text { Windows } 2008 \text { server Datacenter } \\
\text { x86 SP1 (kernel as Windows Vista } \\
\text { ultimate sp2) }\end{array}$ & 172.23 .202 .117 & 3 & 2 & 2 & 7 & 3 & 2 & 2 & 7 \\
\hline 18. & $\begin{array}{l}\text { Windows } 2008 \text { enterprise x64 serv- } \\
\text { er R2 SP1 update June } 2011 \text { (kernel } \\
\text { as Windows 7) }\end{array}$ & 172.23 .202 .118 & 0 & 2 & 2 & 4 & 0 & 2 & 2 & 4 \\
\hline 19. & Windows 7 Home Basic SP1 x64 & 172.23.202.100 & 0 & 2 & 2 & 4 & 0 & 2 & 2 & 4 \\
\hline 20. & Windows 7 Home Basic SP1 x86 & 172.23.202.99 & 0 & 2 & 2 & 4 & 0 & 2 & 2 & 4 \\
\hline 21 & $\begin{array}{l}\text { Windows } 7 \text { Home Premium SP1 } \\
\text { x64 }\end{array}$ & 172.23.202.98 & 0 & 2 & 2 & 4 & 0 & 2 & 2 & 4 \\
\hline 22. & $\begin{array}{l}\text { Windows } 7 \text { Home Premium SP1 } \\
\text { x86 }\end{array}$ & 172.23.202.97 & 0 & 2 & 2 & 4 & 0 & 2 & 2 & 4 \\
\hline 23. & Windows 7 Professional SP1 x64 & 172.23.202.96 & 0 & 2 & 2 & 4 & 0 & 2 & 2 & 4 \\
\hline 24. & Windows 7 Professional SP1 x86 & 172.23.202.95 & 0 & 2 & 2 & 4 & 0 & 2 & 2 & 4 \\
\hline 25. & Windows 7 starter & 172.23.202.94 & 0 & 2 & 2 & 4 & 0 & 2 & 2 & 4 \\
\hline 26. & Windows 7 Professional N x64 & 172.23.202.93 & 0 & 2 & 2 & 4 & 0 & 2 & 2 & 4 \\
\hline 26. & Windows 7 Professional N x86 & 172.23 .202 .92 & 0 & 2 & 2 & 4 & 0 & 2 & 2 & 4 \\
\hline 28. & Windows 7 Professional x64 & 172.23.202.91 & 0 & 2 & 2 & 4 & 0 & 2 & 2 & 4 \\
\hline
\end{tabular}




\begin{tabular}{|c|c|c|c|c|c|c|c|c|c|c|}
\hline \multirow[t]{2}{*}{ No. } & \multirow[t]{2}{*}{ Operating system } & \multirow[t]{2}{*}{ IP Address } & \multicolumn{4}{|c|}{$\begin{array}{c}\text { Number of } \\
\text { vulnerabilities in } \\
2011 \\
\end{array}$} & \multicolumn{4}{|c|}{$\begin{array}{c}\text { Number of } \\
\text { vulnerabilities in } \\
2013 \\
\end{array}$} \\
\hline & & & $\mathrm{Cr}$ & Se & Mo & To & $\mathrm{Cr}$ & Se & Mo & To \\
\hline 29. & Windows 7 Professional x86 & 172.23.202.90 & 0 & 2 & 2 & 4 & 0 & 2 & 2 & 4 \\
\hline 30. & Windows Vista Home Basic x86 & 172.23.202.89 & 4 & 2 & 2 & 8 & 4 & 2 & 2 & 8 \\
\hline 31. & Windows Vista Home Basic x64 & 172.23.202.88 & 4 & 2 & 2 & 8 & 4 & 2 & 2 & 8 \\
\hline 32. & Windows Vista Home Premium x86 & 172.23 .202 .87 & 4 & 2 & 2 & 8 & 4 & 2 & 2 & 8 \\
\hline 33. & Windows Vista Home Premium x64 & 172.23 .202 .86 & 4 & 2 & 2 & 8 & 4 & 2 & 2 & 8 \\
\hline 34. & Windows Vista Business x86 & 172.23.202.85 & 4 & 2 & 2 & 8 & 4 & 2 & 2 & 8 \\
\hline 35. & Windows Vista Business x64 & 172.23.202.84 & 4 & 2 & 2 & 8 & 4 & 2 & 2 & 8 \\
\hline 36. & Windows Vista Ultimate x64 & 172.23.202.83 & 4 & 2 & 2 & 8 & 4 & 2 & 2 & 8 \\
\hline 37. & $\begin{array}{l}\text { Windows Vista Home Basic x86 } \\
\text { SP2 }\end{array}$ & 172.23 .202 .82 & 3 & 2 & 2 & 7 & 3 & 2 & 2 & 7 \\
\hline 38. & $\begin{array}{l}\text { Windows Vista Home Basic x64 } \\
\text { SP2 }\end{array}$ & 172.23 .202 .81 & 3 & 2 & 2 & 7 & 3 & 2 & 2 & 7 \\
\hline 39. & Windows Vista Business x86 SP2 & 172.23 .202 .80 & 3 & 2 & 2 & 7 & 3 & 2 & 2 & 7 \\
\hline 40. & Windows Vista Business x64 SP2 & 172.23.202.79 & 3 & 2 & 2 & 7 & 3 & 2 & 2 & 7 \\
\hline 41. & $\begin{array}{l}\text { Windows Vista Home Premium x86 } \\
\text { SP2 }\end{array}$ & 172.23 .202 .78 & 3 & 2 & 2 & 7 & 3 & 2 & 2 & 7 \\
\hline 42. & $\begin{array}{l}\text { Windows Vista Home Premium x64 } \\
\text { SP2 }\end{array}$ & 172.23.202.77 & 3 & 2 & 2 & 7 & 3 & 2 & 2 & 7 \\
\hline 43. & Windows 2000 server Sp4 & 172.23.202.76 & 13 & 4 & 1 & 18 & 14 & 4 & 1 & 19 \\
\hline 44. & $\begin{array}{l}\text { Windows server } 2003 \text { Enterprise } \\
\text { x86 SP1 }\end{array}$ & 172.23 .202 .75 & 10 & 3 & 1 & 14 & 10 & 3 & 1 & 14 \\
\hline 45. & $\begin{array}{l}\text { Windows server } 2003 \text { Standard x86 } \\
\text { SP1 }\end{array}$ & 172.23 .202 .74 & 10 & 3 & 1 & 14 & 10 & 3 & 1 & 14 \\
\hline 46. & $\begin{array}{l}\text { Windows server } 2003 \text { Standard x64 } \\
\text { SP1 }\end{array}$ & 172.23 .202 .73 & 10 & 3 & 1 & 14 & 10 & 3 & 1 & 14 \\
\hline 47. & $\begin{array}{l}\text { Windows Server } 2003 \text { Enterprise } \\
\text { x64 SP2 }\end{array}$ & 172.23 .202 .72 & 6 & 2 & 1 & 9 & 6 & 2 & 1 & 9 \\
\hline 48. & $\begin{array}{l}\text { Windows server } 2003 \text { Standard x86 } \\
\text { SP2 }\end{array}$ & 172.23 .202 .71 & 6 & 2 & 1 & 9 & 6 & 2 & 1 & 9 \\
\hline 49. & $\begin{array}{l}\text { Windows server } 2003 \text { Standard x64 } \\
\text { SP2 }\end{array}$ & 172.23 .202 .70 & 6 & 2 & 1 & 9 & 6 & 2 & 1 & 9 \\
\hline 50. & Windows XP pro sp1 x64 & 172.23.202.69 & 11 & 3 & 1 & 15 & 11 & 3 & 1 & 15 \\
\hline 51 & $\begin{array}{l}\text { Windows } 2008 \text { server Enterprise } \\
\text { x86 SP1 }\end{array}$ & 172.23.202.68 & 3 & 2 & 2 & 7 & 3 & 2 & 2 & 7 \\
\hline \multicolumn{3}{|c|}{$\begin{array}{c}\text { TOTAL } \\
\end{array}$} & 211 & 117 & 86 & 414 & 214 & 120 & 83 & 417 \\
\hline
\end{tabular}

Table 5 Number of vulnerable services on Windows OS classified according to severity 


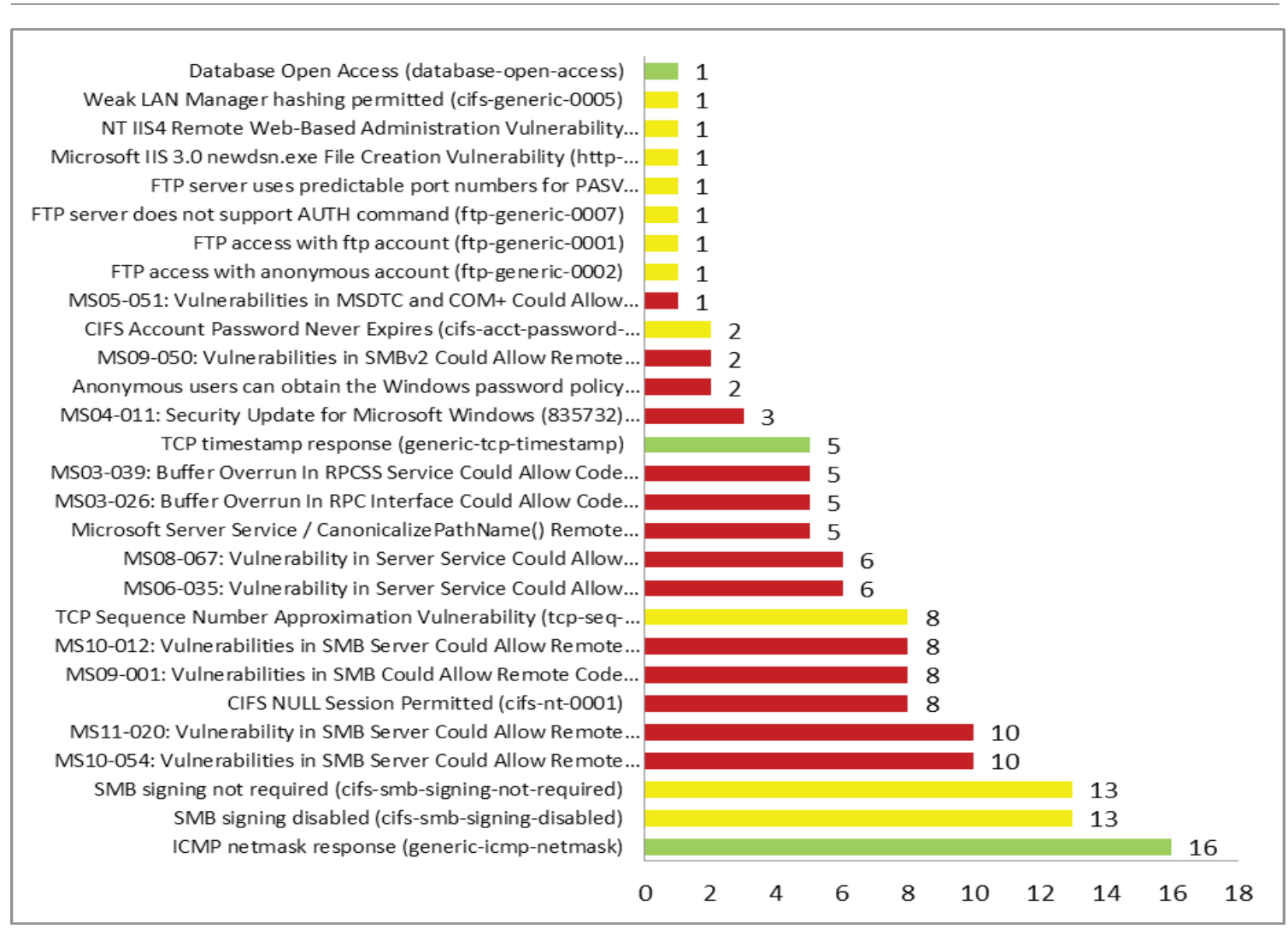

Graph 4 Overview of found vulnerabilities by frequency in tested systems in 2011 for Windows OS

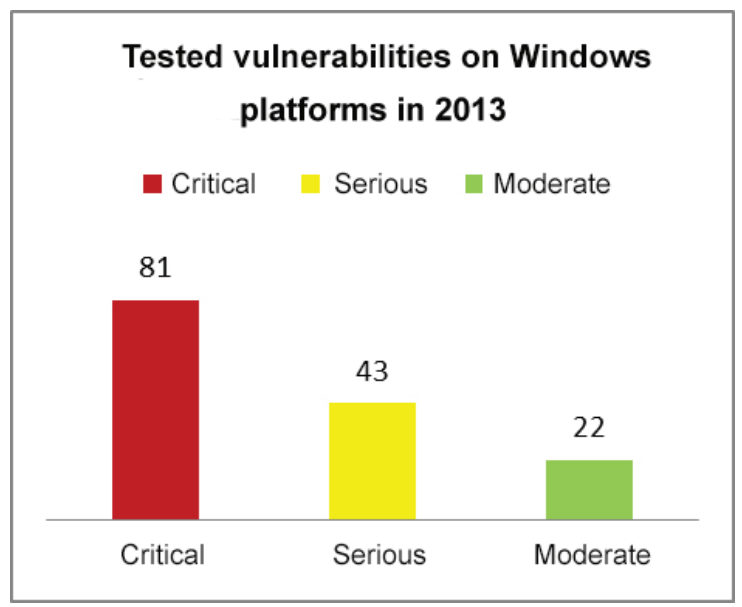

Graph 5. Found vulnerabilities on tested Windows OS in 2013

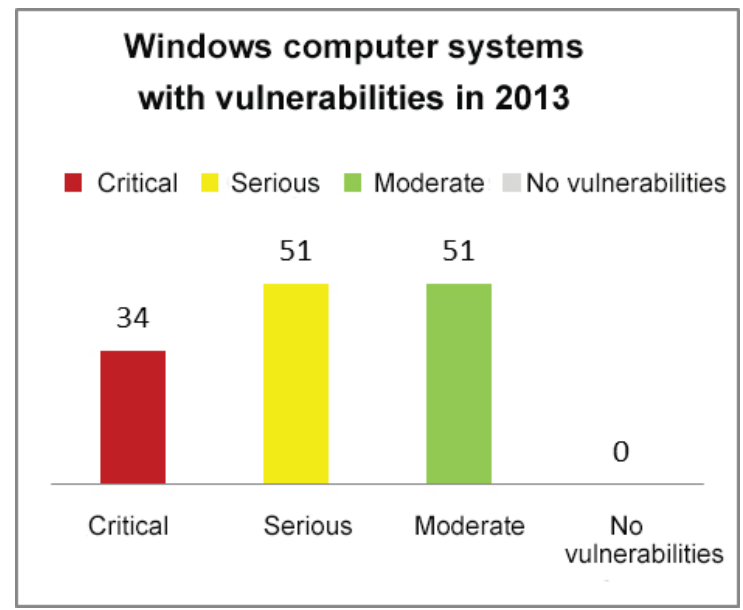

Graph 6 Number of Windows computer systems by severity of vulnerability in 2013
(Graph 3.). Serious vulnerabilities were found in a total of 51 computer systems. Moderate vulnerabilities are also present in 51 operating systems. The presentation of detected vulnerabilities according to frequency in the examined systems in 2011 is given in Graph 4. Although no additional services have been added after the default instal- lation, it can be concluded that none of the tested systems were without vulnerability.

In 2013, 146 unique vulnerabilities were found, and at the level of all scanned Windows systems, the total number is 417 vulnerabilities (Table 5, Graph 1, Figure 3). Out of this number, 81 critical, 43 serious and 22 moderate vulnera- 


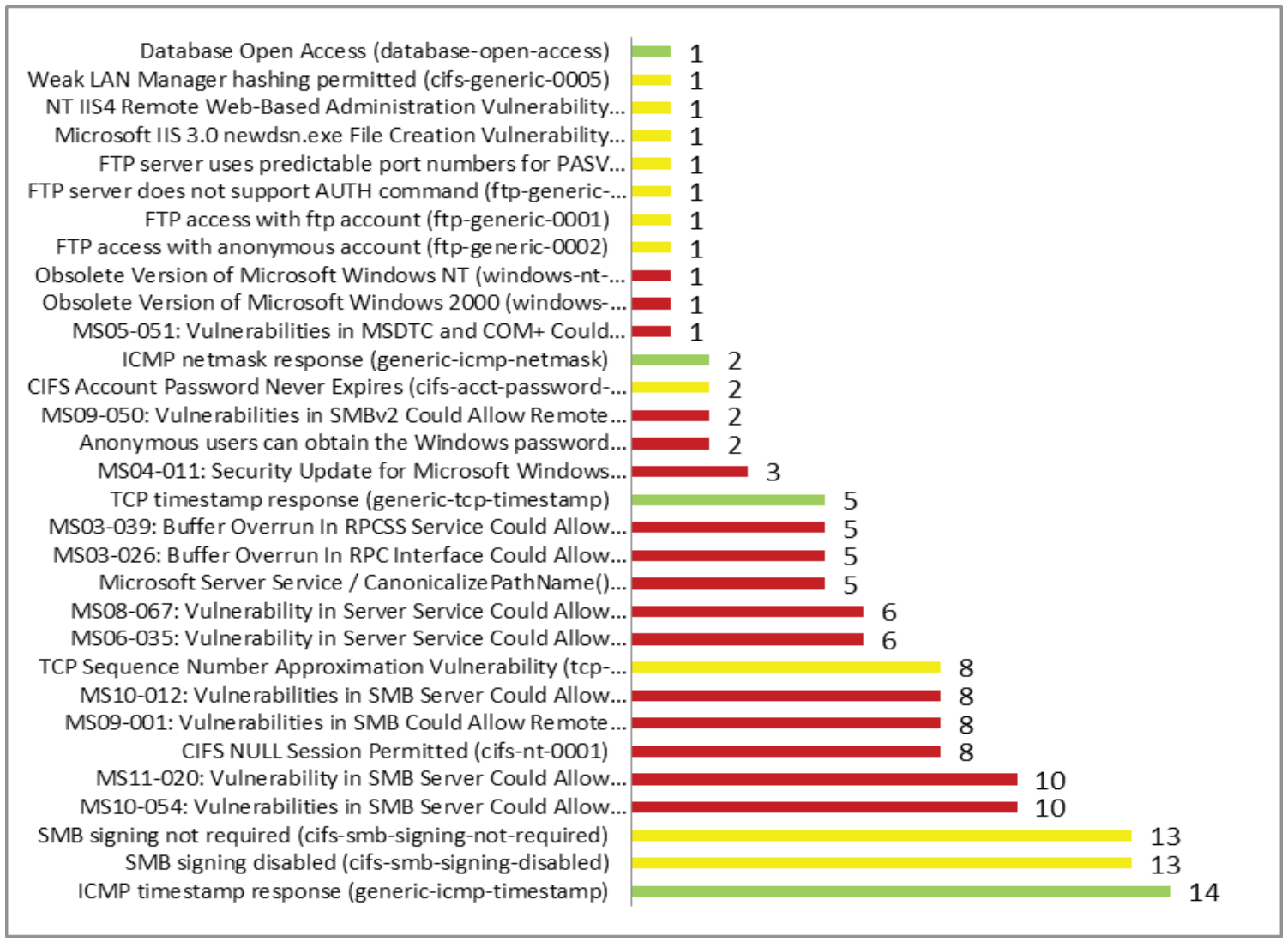

Graph 7 Overview of found vulnerabilities by frequency on tested systems in 2013

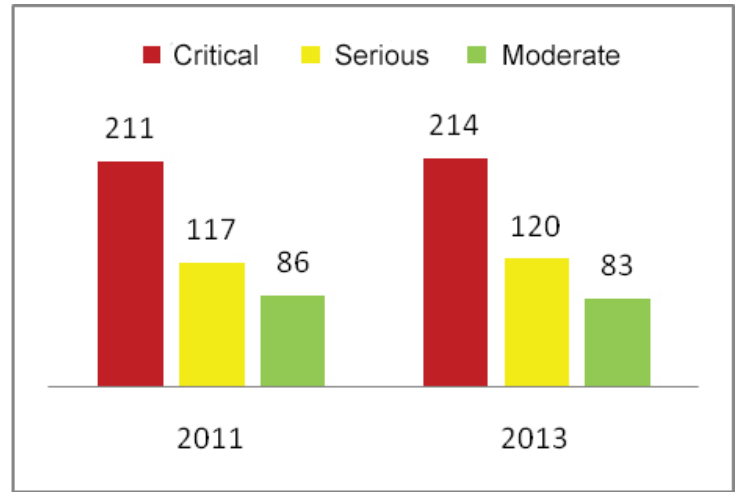

Graph 8 Global overview of vulnerabilities for all Windows operating systems by year

bilities were found (Graph 5). When considering computer systems individually, 214 critical, 120 serious and 83 moderate vulnerabilities were found. Critical vulnerabilities were found on 34 computer systems and they are most susceptible to attack. Serious vulnerabilities were found in 51 computer systems (Graph 6.). Moderate vulnerabilities are also present on 51 operating systems
(Graph 6). The presentation of detected vulnerabilities according to frequency in the examined systems in 2013 is given in Graph 7. Although no additional services have been added after the default installation, it can be concluded that none of the tested systems were without vulnerability.

As presented above it can be concluded that all scanned systems are vulnerable, but there has been no significant increase of vulnerability in the period between 2011 and 2013 (as for the scanned Windows operating systems without installed additional services, see Graph 8). Significant growth of vulnerability sources has been also noted on Windows operating systems from 2646 to 3951 (Table 4, Graph 1). Given that no significant increase of vulnerability has been recorded, while a significant growth of the sources of vulnerabilities has been reported, it can be concluded that the existing vulnerabilities have been misused in various ways. 


\section{CONCLUSION}

The importance and development of new technologies for business modernization and data transfer are constantly increasing. Unfortunately, illegal activities are spreading at the same time. The problem of computer crime is a complex phenomenon. Since the perpetrators of such acts have the necessary knowledge and use sophisticated techniques for their execution, it is all the more difficult to trace and undoubtedly prove the elements of the criminal offence.

The vulnerability scanning of Windows operating systems has been performed with the Rapid 7 Nexpose tool. The aim of this experimental research is actually twofold. On the one hand, vulnerable services that can endanger the security of the system are presented, and on the other, it is possible to apply adequate proactive protection measures based on the recognized vulnerabilities. It has been confirmed that after default installations there is no computer system without vulnerability.

The total number of scanned Windows operating systems is 51 . In this way, the vulnerabilities of Windows operating systems installed by default are presented, with an aim to indicate potential security vulnerabilities, as well as adequate preventive measures for system protection.

With proper and regular use of tools for scanning and logging vulnerabilities on systems, it is possible to get detailed insight into illegal processes in the system and to prevent further illegal activities within a network or a particular computer system. By integrating the results of proactive digital forensics together with systems of preventive protection, detection and analysis of vulnerability, as well as by implementing multilayer protection architecture (Korać 2010), with timely response to incidental or illegal activities (with a digital forensics specialist), it is possible to increase system security and achieve an optimal level of protection which is suitable to a defined security policy.

Since this topic covers vulnerability scanning technology on Windows operating systems, this work is exceptionally applicable and useful for researchers, students in these fields, computer system administrators, legal and social experts, as well as experts in criminal justice.

\section{BIBLIOGRAPHY}

\section{Grubor, G. and Gotić, A. 2012}

Korporativna aktivna digitalna forenzička istraga primenom Backtrack - a, 10. Međunarodni naučni skup Sinergija 2012. Univerzitet Sinergija, 2012.

\section{Korać, V. 2010}

Infrastruktura sa javnim ključevima u funkciji zaštite informacionog toka i elektronskog poslovanja, Arheologija i prirodne nauke, specijalna izdanja, Centar za nove tehnologije, 2010.

\section{Korać, V. 2014}

Digitalna forenzika u funkciji zaštite informacionog sistema baziranog na Linux $i$ Windows platformama, unpublished doctoral thesis, Univerzitet u Beogradu, 2014.

\section{REZIME \\ ISPITIVANJE WINDOWS DIFOLT- NIH SERVISA NA RANJIVOSTI}

Upotrebom alata za analizu ranjivih servisa na sistemu moguće je dobiti dragocene informacije o sistemu i mreži sa stanovišta zaštite. Istraživanjem je obuhvaćeno 51 Windows operativnih sistema. Prikupljene informacije obuhvataju veliki broj podataka o prisustvu različitih mrežnih servisa na sistemu koji predstavljaju potencijalne bezbednosne propuste. Na taj način su prezentovane, ranjivosti difoltno instaliranih Windows operativnih sistema sa ciljem ukazivanja na potencijalne bezbednosne ranjivosti. Ove ranjivosti odnosno propusti mogu nastati zbog pogrešno konfigurisanih servisa, poznatih grešaka (eng. Well known bug) u sistemu ili programu, neažuriranosti sistema i njegovih servisa, kao i zbog upotrebe slabe zaš- 
tite u konfiguraciji. Cilj ovog ispitivanja jeste da se identifikuju i na osnovu toga koriguju svi prepoznati bezbednosni propusti (ranjivi servisi) na difoltno instaliranim Windows sistemima. 